\title{
Multi-Target C Code Generation from MATLAB ${ }^{\circledR}$
}

\author{
João Bispo, Luís Reis \\ Faculty of Engineering (FEUP) \\ University of Porto, Porto, Portugal \\ \{jbispo, ei09030\}@fe.up.pt
}

\author{
João M. P. Cardoso \\ Faculty of Engineering (FEUP) \\ University of Porto \& INESC-TEC, Porto, Portugal \\ jmpc@fe.up.pt
}

\begin{abstract}
This paper describes our recent work on MATISSE, a framework for MATLAB to C compilation. We focus on the new optimizations and transformations, as well as on OpenCL generation. MATISSE is controlled with LARA, an aspect-oriented language, able to specify transformations to the input MATLAB code (e.g., insertion of code for variable initialization and for monitoring) and to express information concerning types and shapes of variables. We evaluate the compiler with a set of benchmarks when targeting both an embedded system and a desktop system. The results show that we were able to achieve a speedup up to $1.8 \times$ by employing information provided by LARA aspects. We also compare the execution time of the generated $\mathrm{C}$ code with the original code running on MATLAB, and we achieve a geometric mean speedup of $19 \times$. The geometric mean speedup reduces to $12 \times$ when optimizing the MATLAB code with LARA aspects. Finally, we present a preliminary version of a fully-functioning pragma-based OpenCL generator, built over the MATISSE framework.
\end{abstract}

Categories and Subject Descriptors D.3.4 [Programming Languages]: Processors - Code generation, Compilers, Optimization, Retargetable compilers D.2.2 [Software Engineering]: Design Tools and Techniques

General Terms Performance, Experimentation, Languages

Keywords MATLAB-to-C, source-to-source compiler, Aspectoriented programming, LARA, embedded systems, OpenCL

\section{Introduction}

$M A T L A B$ [1] is a de facto standard high-level programming language and interactive numerical computing environment in many domains in engineering and science, including embedded computing as it is ubiquitously used by engineers to quickly develop and evaluate their solutions. MATLAB is dynamically typed, and relies on interpretation (and/or JIT compilation) as the information about the types and shapes (i.e., number and size of matrix dimensions) of variables is only known at runtime. Due to advances in JIT compilation and the use of pre-compiled libraries for the most intensive functions, the MATLAB runtime environment currently exhibits acceptable performance.

Permission to make digital or hard copies of all or part of this work for personal or classroom use is granted without fee provided that copies are not made or distributed for profit or commercial advantage and that copies bear this notice and the full citation on the first page. Copyrights for components of this work owned by others than ACM must be honored. Abstracting with credit is permitted. To copy otherwise, or republish, to post on servers or to redistribute to lists, requires prior specific permission and/or a fee. Request permissions from Permissions@acm.org. ARRAY' 14, June 11 2014, Edinburgh, United Kingdom Copyright 2014 ACM 978-1-4503-2937-8/14/06 \$15.00. http://dx.doi.org/10.1145/2627373.2627389
In many embedded system settings, however, the use of a $M A T L A B$ runtime environment is infeasible, either because it is not available, or due to performance and/or resource constraints. To address this potential shortcoming, a typical solution relies on the development of an implementation in executable code written in an imperative language such as $\mathrm{C} / \mathrm{C}++$ once the base or original $M A T L A B$ code has been validated. This implementation must then in turn be validated against the output of the MATLAB code resulting on a lengthy and error prone process that further complicates the overall application development cycle and cost. The existence of two code specifications - the original prototypical MATLAB code and the reference $\mathrm{C} / \mathrm{C}++$ code - also exacerbates maintenance costs. Another solution is to rely on the automatic translation of MATLAB to the target programming language as provided, e.g., by the MATLAB Coder [2] and the Embedded Coder [3], which translate MATLAB to $\mathrm{C}$ code. Besides the inherent advantages, this, however, has typically the disadvantage of the low support to control and guide the code translation. The code generation is typically based on directives (GUI based in the case of the MATLAB Coder) addressing types, shapes, and target. When dealing with the myriad of target architectures and toolchains in embedded systems, this approach presents a low level of flexibility, e.g., as the style of the $\mathrm{C}$ code generator might need to be tuned to the toolchain as is the case when targeting $\mathrm{C}$ to hardware compilers. Instrumentation and code transformations, as well as an approach to express strategies for code transformations and instrumentation (e.g., by using a DSL) can be very important during the design process and may increase productivity. Furthermore, the target platform may require code generators to specific programming languages as is the case of the generation of OpenCL [4] when dealing with GPGPUs and/or GPU-based FPGA implementations [5].

Our approach relies on a compilation tool, named as MATISSE [6], which generates C code directly from MATLAB. Our approach explores the use of Aspect-Oriented Programming (AOP) [7][8] concepts, through the use of the LARA language [9][10] as a vehicle to convey information to the compiler (e.g., types and array shapes) and to express code specialization and code instrumentation strategies. The compiler uses the userprovided information complementing and checking its consistency against the information it can derive from its own analysis. MATISSE is being developed as a modular and flexible compiler framework, which includes custom Intermediate Representations (IRs) for the MATLAB and C code, keeping in mind the generation of $\mathrm{C}$ code from a higher-level programming language. In particular, the IR representing the output $\mathrm{C}$ code (C-IR) supports matrix types natively, and can be easily extended to support additional types and language constructs (such as the ones needed to generate OpenCL). The end result is a synergy between compiler analysis and the user that allows the compiler to generate very high-quality code from MATLAB specifications. It is also possible 
to generate different versions of the $\mathrm{C}$ code, to better target different embedded systems, platforms, and/or toolchains. In this paper we focus on our recent MATISSE improvements.

The remainder of this paper is organized as follows. Section 2 presents the MATISSE compiler framework. In Section 3 we describe the $\mathrm{C}$-IR, the internal representation we use to generate $\mathrm{C}$ code. Section 4 explains how the MATLAB code is transformed into $\mathrm{C}$ code and OpenCL with the help of LARA aspects. Section 5 shows some experiments performed using MATISSE. Section 6 describes related work and finally, Section 7 concludes this paper and describes ongoing work.

\section{MATISSE Overview}

MATISSE consists of a MATLAB-to-C compiler targeting embedded systems, and a LARA-controlled MATLAB weaver which allows transformations over MATLAB code. LARA [9] is a domain-specific language inspired by AOP concepts [7] and JavaScript semantics and constructs. LARA uses a declarative semantic to allow programmers to specify strategies for actions over application source code and/or compiler IRs (e.g., instrument code, extract information, explore transformations, apply compiler optimizations). LARA aspects are applied by a target language dependent weaver, such as the weaver for the MATLAB language integrated in MATISSE.

Figure 1 presents the overall flow of MATISSE. The input MATLAB files are parsed and translated to an abstract syntax tree (AST) based MATLAB IR. The IR is the input to MWeaver, which uses LARA aspects to modify and add information (e.g., variable types and shapes) to the MATLAB IR. MATISSE is being developed in a way to make easy the integration of code generators. At the moment code generators for MATLAB and C are already fully working and an OpenCL code generator is under development.

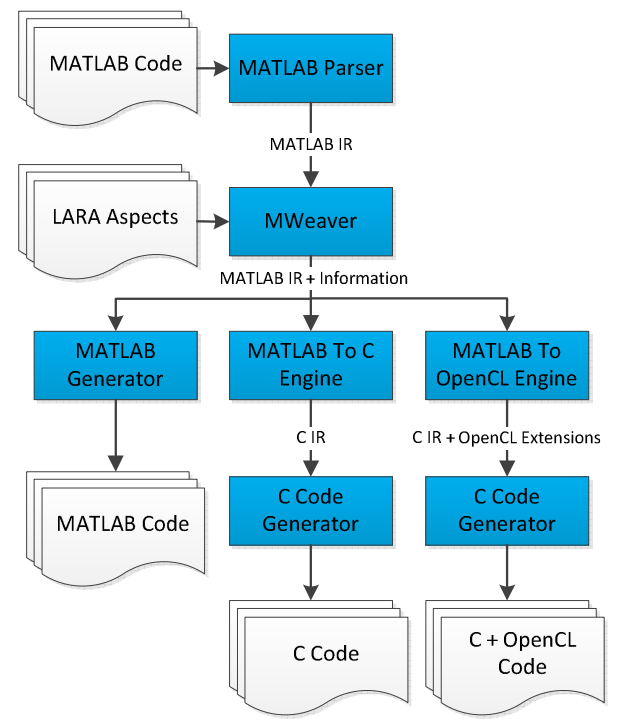

Figure 1. Overview of the MATISSE compiler framework.

MATISSE generates MATLAB code for validation, testing, monitoring, and specialization, and $\mathrm{C}$ code to be used by thirdparty design-flows targeting software/hardware systems. MATISSE is able to generate customized $\mathrm{C}$ code for a particular target without modifying the original MATLAB code. LARA aspects enable MATISSE to have fine-grained control over the generation of $\mathrm{C}$ code, and also allow the generation of different implementations from the same source code. A common example includes the restructuring of source code and the use of statically declared array variables to be compliant with the requirements of most hardware compilers.

MATISSE can be used as a source-to-source code transformation and instrumentation tool allowing developers to quickly and reliably generate reference $\mathrm{C}$ implementations, a key step in the deployment of embedded system applications. The transformation stage of the compiler performs weaving actions such as insertion of code, definitions of types and shapes, and code specialization based on default values.

\section{A High-Level C-IR}

Given the differences between MATLAB and C, we decided to use a $C$ specific AST-based IR to represent the $C$ code (C-IR). This simplifies the generation of $\mathrm{C}$ code and allows us to separate concerns related to $\mathrm{C}$ generation (e.g., include files, variable declarations) that are not considered in the MATLAB IR. This option also makes the MATLAB IR clean and independent of the specificities of the code generation to be applied.

\subsection{Variable Types}

The C-IR uses the VariableType interface to represent all the information needed about a data type (e.g., code needed to declare the variable, how to convert to another type, how to perform an addition between variables of this type). VariableType allows a seamless integration of several types in a modular way. For instance, we were able to add support for OpenCL native types through an implementation of VariableType, without changing the C-IR library. Figure 2 shows a subset of the hierarchy that starts with the VariableType interface. To add a new type, one needs to create a class that implements the VariableType interface. Scalar represents a single value, and Matrix represents a multidimensional array of elements of type Scalar. These two classes add contract methods that provide information specific to these types, such as number of bits, maximum and minimum values or signed/unsigned, for Scalar, or matrix shape and element type, for Matrix. CNative represents the native types of C (e.g., int, float, in the case of Numeric, and, e.g., int32 t, uint $\_t$ in the case of StdInt).

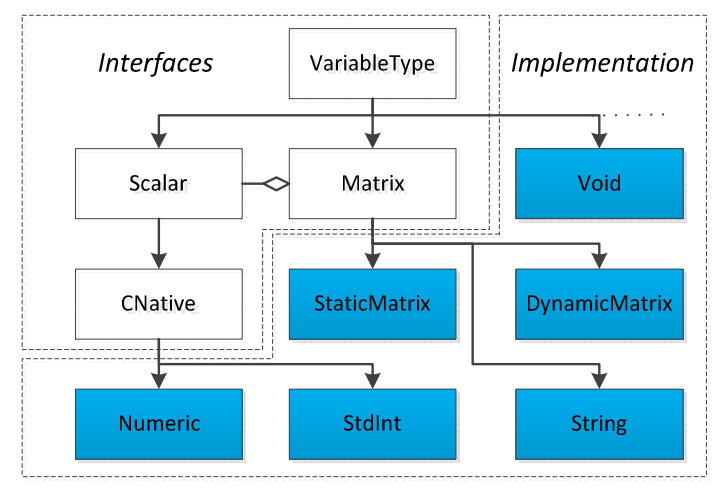

Figure 2. Subset of the VariableType hierarchy in the C-IR.

\subsection{Matrix Types in C-IR}

Currently, the C-IR has two Matrix implementations, StaticMatrix, which uses statically allocated $\mathrm{C}$ arrays (e.g., int $A[3]$ ), and DynamicMatrix, which uses a C structure to represent dynamically allocated arrays of a given element type. For dynamic arrays, MATISSE allows the option to use for each array access a func- 
tion that performs array bounds checking (for debugging), or to use inlined code without checks (for performance).

MATISSE uses linearization of multi-dimensional arrays, whereby an element of the multi-dimensional array is accessed through a single pointer variable. Linearization has several benefits. Firstly, simple element-wise operations are compactly executed in a single loop rather than using a loop nested structure. Secondly, the single allocation of storage and corresponding boundary values also enables one out-of-bound condition check per array access rather than having to perform a verification per array dimension. Lastly, it also provides other advantages regarding the size of the code generated. When multi-dimensional arrays are generated without linearization, code may need to consider various pointers (when dimensions are not known at compilation time) in order to allocate the space needed for all dimensions.

\section{Transforming MATLAB to $\mathrm{C}$}

MATLAB is a dynamically typed language. This is in stark contrast with $\mathrm{C}$, which is statically typed and needs the types of all variables to be declared. When converting MATLAB to efficient $\mathrm{C}$ code, it is necessary to statically determine the types used. This can be a challenge, as the same MATLAB function can have very different $\mathrm{C}$ implementations, depending on the types of its variables. Fortunately, defining the types of the arguments/parameters of a MATLAB function is often enough to infer the remaining types of variables in the function. In the case of static arrays, usually it is also possible to determine the shape of the arrays at compile time (i.e., how many dimensions the array has, as well as the size of each dimension).

MATISSE uses an interface that represents all possible $\mathrm{C}$ implementations for a given named MATLAB function and selects one of them based on the received arguments and types. The specialization occurs at the level of the function call, instead of the function. This mechanism allows MATISSE to generate multiple versions of highly specialized $\mathrm{C}$ functions. For instance, if there are multiple function calls to a MATLAB function that accepts a matrix type as input, and it receives different StaticMatrix types (a type whose shape is known at compile time), it is possible to generate a function specialized to each specific shape.

\subsection{Type and Shape Inference Analysis}

The type inference uses a simple data-flow analysis approach [11], where type information is derived by processing each MATLAB statement, complemented with information provided by LARA aspects. In most cases only the types of function parameters are required for the compiler to achieve an efficient type inference. There are two general situations where type-inference is applied in MATISSE: 1) during a function call; and 2) during assignments. C-IR nodes representing function calls contain information about the signature of the functions (i.e., their input and output types), and are specialized according to the inputs of the call. The output types are usually determined by the object that creates the function call, and this means that each function can define its own rules regarding the type-inference of its output types. For the assignments, the variables on the left hand are usually bound to the type inferred in the right hand. Note that when in conflict, types of variables defined in a LARA aspect always override the inference mechanism. Besides the type, variables can carry other information, such as values and the shapes of matrices. This information is propagated and in many cases updated and extended by information determined in other assignments.

Consider the code in Figure 3. In the first line, the function size returns an array with the shape of the given variable. If the varia- ble $H$ is of the type StaticMatrix, it always contains information about its shape, and the values of $h 1$ and $h 2$ are known at compile time. If $H$ is a DynamicMatrix, the values of $h 1$ or $h 2$ might not be known. Size is a supported MATLAB function, and MATISSE creates a FunctionCall node in C-IR, specialized to the type of $H$. C-IR nodes always carry information about the types they return. Thus, MATISSE assigns the types defined by the FunctionInstance of size to $h 1$ and $h 2$ (in this case, both are of type int). However, if the types of $h 1$ and $h 2$ are defined in a LARA aspect, MATISSE uses those types. If the shape of $H$ is known at compile time, the values of $h l$ and $h 2$ are also known.

In the second line in Figure 3, for operations such as + there is a default rule that chooses the first maximal fit type between the operands. The maximal fit is automatically determined using information obtained from the Scalar interface (i.e., the minimum and maximum possible values of the type). Although MATISSE infers types for constants (e.g., 1 is inferred as an integer, 1.0 as a double), for this rule the types of constants are not taken into account when inferring the type of the output (unless all operands are constants). In this case, the result of the addition will have the same type as $h l$. The operator / has special inference rules. By default, the output is assigned to a real type, to avoid losing precision. The flexibility of MATISSE allows, on the one hand, general rules available to all functions, and on the other hand, custom rules for a particular function. As previously referred, if a LARA aspect defines the type of any of the variables (i.e., $H, h 1, h 2$ or offset 1 ) the inference mechanism for that particular type is augmented with that information. For instance, if the type of the variable offset 1 is set to float, the operations on the right-hand of the assignment will also consider float as output. This way it is possible to address the limitations of a static type/shape inference analysis, and the usual cases where the user needs to force and evaluate data types not derived by type inference.

$\begin{aligned} {[\mathrm{h} 1, \mathrm{~h} 2] } & =\operatorname{size}(\mathrm{H}) \\ \text { offset } 1 & =(\mathrm{h} 1+1) / 2\end{aligned}$

Figure 3. Code snippet from conv2.

\subsection{Code Generation Example}

We now illustrate the application of the proposed approach to a $M A T L A B$ function implementing an FIR (Finite Impulse Response) filter (see Figure 4). This function takes as input two arrays, vector_ld and coef, and outputs an array named output. In the absence of information about the shape of input arrays, MATISSE generates $\mathrm{C}$ code that uses the DynamicMatrix type for the parameter as well as for the function's return value, which represents a structure with dynamically allocated memory. The definition of types and shapes for the function parameters in a LARA aspect (see Figure 5) enables the use of the StaticMatrix type.

The MATLAB code in Figure 4 uses the MATLAB built-in function sum. Currently, MATISSE supports a general version of sum, by using a description in MATLAB translated to C by MATISSE. With information about the types of the arguments of sum, we can apply transformations over the code. Figure 6 shows a possible transformation, which is applied as follows. The function sum is called with an expression as argument. By analysing the MATLAB-IR corresponding to the expression, MATISSE determines that it is composed only by element-wise operations (i.e., . $\left.{ }^{*}\right)$. Furthermore, the operands in the expression return onedimension arrays (coef is used directly and we know the shape, and vector $1 d$ is accessed linearly, returning a one-dimension array). The output of sum will be a scalar, and can be replaced with an accumulator variable (sum_acc) and a for loop. If there 
were no ranges, we could iterate the loop over the size of any of the input matrices. As there is a range, we use it to control the for loop: (i:-1:i-NTAPS+1). If there were any other ranges, MATISSE would calculate the indexes outside the for and would access them with an induction variable. The matrix coef is used as parameter, so the compiler uses an induction variable (matrix $i$ ) to access the elements of coef and incremented after each loop iteration. This is an example of a transformation applied with the current framework, at the MATLAB-IR level. In this case, the output of the transformation is a modified MATLAB-IR, but we can perform the same transformation to generate directly C-IR. This transformation in particular will slow down the code if executed in MATLAB, but can significantly help to generate efficient $\mathrm{C}$ code (see Figure 7).

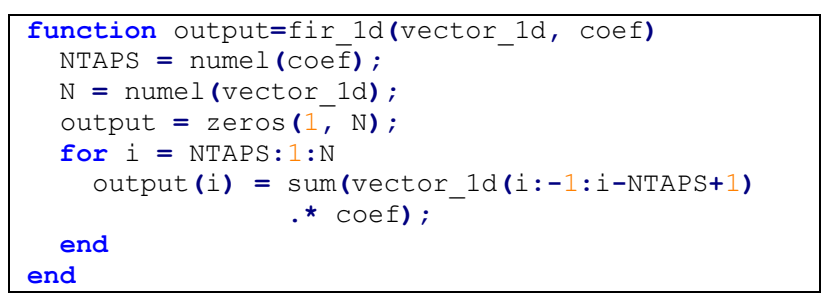

Figure 4. MATLAB fir code example.

\begin{tabular}{|c|}
\hline $\begin{array}{l}\text { aspectdef firSingle } \\
\text { var typeDef }=\{/ / \text { Type definition } \\
\text { vector_ld : "single[1][1024]", } \\
\text { coefficients : "single[1][32]", } \\
\text { output : "single" \}; } \\
\text { // Matrix sizes } \\
\text { var matrixSizes = } \text { output : "1, N" \}; } \\
\text { // Define types } \\
\text { call defineTypes("fir_1d", typeDef); } \\
\text { // Define matrix sizes } \\
\text { call initMatrixes("fir_1d", matrixSizes); } \\
\text { // Inline all functions MATISE supports } \\
\text { call matisseInline("true"); } \\
\text { // Define the matrix implementation as static } \\
\text { call matisseMatrixImpl("static") } \\
\text { end }\end{array}$ \\
\hline
\end{tabular}

Figure 5. LARA aspect that defines the types for the fir example.

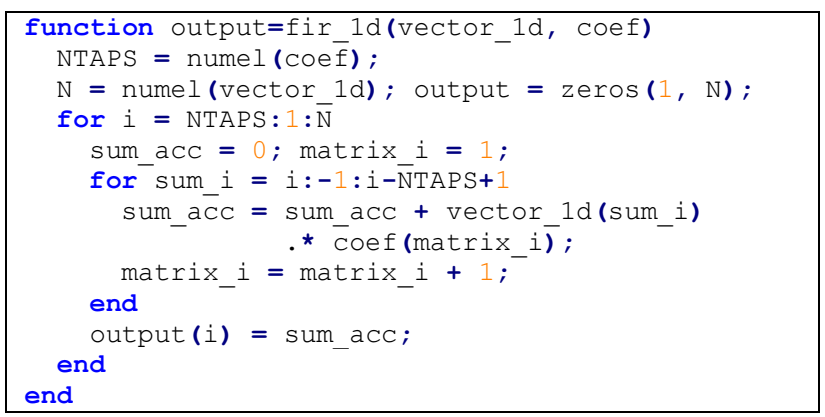

Figure 6. MATLAB fir after transformation.

\subsection{OpenCL Generation}

We are developing an OpenCL generator which uses OpenACC [12] based pragmas in the MATLAB code to decide what/how to parallelize. Sections to parallelize begin with the "parallel loop" pragma. The "end" pragma indicates the end of the code section the pragma applies to. Parallel loop sections can be parameterized with copyin, copyout and/or reduce. Two types of reductions are supported: sums and products.
We present the OpenCL engine to illustrate the overall flexibility of the framework, and of the C-IR. In a few months' work, a single Master student was able to develop an OpenCL engine capable of generating the OpenCL code for sections in a relevant subset of MATLAB annotated with pragmas, plus the necessary wrapper classes that perform the communication between the $\mathrm{C}$ and the OpenCL code (both codes are represented using the CIR). The OpenCL engine replaces pragma occurrences with a call to a custom function. It then translates that function to OpenCL.

The OpenCL generation is still in a very early phase and the currently generated code is not optimized.

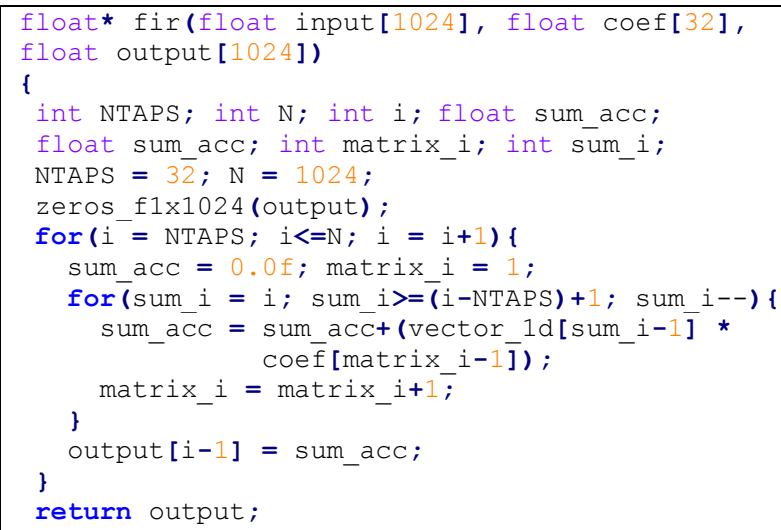

Figure 7. C code with static matrices for the fir function generated by MATISSE with information about shapes.

\section{Experimental Results}

We carried out a series of experiments to evaluate the impact of the information introduced by aspects on the performance of the generated $\mathrm{C}$ code, when executed on an embedded platform. We also compared the execution time of the generated code against the original MATLAB code, when using a desktop PC. Finally, we show some preliminary results obtained by an early version of the OpenCL generator.

\subsection{Methodology}

We use MATISSE to automatically derive $\mathrm{C}$ code corresponding to kernels written in MATLAB. We then compare the execution time after compiling the resulting code to two architectures: 1) A desktop PC with a $2.93 \mathrm{GHz}$ Core 2 Duo processor and Windows 7 32-bit, 3GB of RAM and an nVidia Quadro NVS-290; 2) A BeagleBoard-XM revB running Ubuntu 12.10 32bit, with a $1 \mathrm{GHz}$ ARM Cortex-A8 and $512 \mathrm{MB}$ of RAM.

We use as benchmarks a set of kernels we consider relevant for embedded systems. We include subband and grid it, two critical functions from the $3 D$ Path Planning and the MPEG audio encoder applications [10]. In addition, we include an application to perform correlation using FFTs and with $3 \mathrm{D}$ matrices as input. This application named $c f d$, uses forward and inverse 2D FFTs provided by a MATLAB function able to perform $\mathrm{N}$-dimensional FFTs (identified as $f f t 2 d$ ), and a dot product between 3D matrices (identified as $c p x$ ). Table 1 contains all the benchmarks used.

For both architectures, the generated $\mathrm{C}$ code was compiled with gec-4.6, using flag $-\mathrm{O} 2$ and the OpenCL code was compiled with the AMD APP SDK v2.9. Besides the code of the functions, MATISSE also generates main functions for testing purposes, when specifying a .M or .MAT file with the values of the input arguments of the function to test. All benchmarks output correct 
results when compared with MATLAB original output, up to an error of $10 \mathrm{E}-6$

Table 1. Benchmark characteristics.

\begin{tabular}{|l|r|r|}
\hline \multicolumn{1}{|c|}{ Benchmark } & \multicolumn{1}{c|}{ Input Sizes } & MATLAB LoCs \\
\hline \hline cfd & $256 \times 256 \times 3$ & 50 \\
\hline conv & $96 \times 11$ & 73 \\
\hline cpx & $512 \times 512 \times 10$ & 23 \\
\hline dilate & $2048 \times 2048,2$ & 17 \\
\hline fft2d & $256 \times 256$ & 124 \\
\hline fir1d & $1 \mathrm{M} \times 32$ & 14 \\
\hline grid_it & $32 \times 64 \times 16$ & 38 \\
\hline latnrm & $32 \mathrm{~K} \times 8$ & 29 \\
\hline subband & $128 \times 64 \mathrm{ki}$ & 35 \\
\hline
\end{tabular}

\subsection{Results}

We consider two options for generating C code: aspects (minimal/optimized) and function inlining (enabled/disabled). Minimal aspect refers to the minimum information we have to provide to generate $\mathrm{C}$ code that outputs correct results (sometimes we have to define the type of the output, or of some intermediate variables), while optimized is an aspect that has been tailored to provide specialized code.

Figure 8 shows the impact on performance by each option. Function inlining provides the highest speedups, on average a speedup of $1.6 \times$ for the tested codes (with speedups up to $2.2 \times$ and 2.6×, for grid_it and conv, respectively). This was expected as inlining removes the overhead of calling a function and enables further optimizations. In the case of the inlining of get/set functions, we are trading array-bound checking for performance. Specialization achieved a more modest effect, on average a speedup of $1.2 \times$, with a maximum of $1.8 \times$ for subband. When specializing with aspects, we are replacing certain types with potentially less expensive types (doubles with floats, floats with integers), and reducing the overhead of type casting. In architectures more sensitive to data types a larger impact is expected (e.g., when double precision is supported by software).

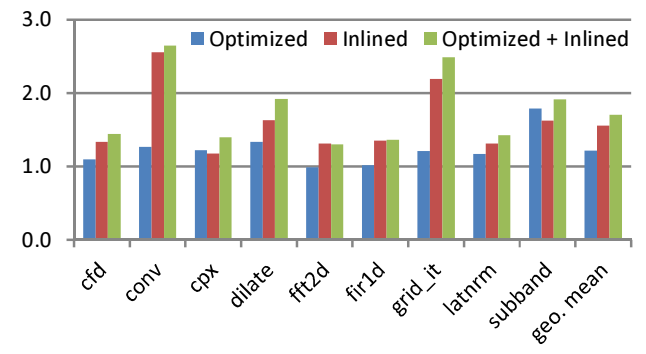

Figure 8. Speedups on the BeagleBoard of the $\mathrm{C}$ code when considering three options.

Figure 9 presents the speedups of the $\mathrm{C}$ code generated by MATISSE when compared over the execution of the original MATLAB code when using MATLAB 2012b, on the Core 2 Duo. Significant speedups were achieved for most cases, ranging from $5 \times$ to $9 \times$. We measured a slowdown of $0.6 \times$ for $f f t 2 d$. The slowdown is related to missing opportunities for optimizing the code of the function. We also achieved very high speedups. E.g., for $c p x$, the main reason is the pre-allocation of matrices inserted by the LARA aspect. If we compare to the execution time in MATLAB after the code is transformed according to LARA specifications, the speedup reduces from $105 \times$ to $5 \times$ (the geometric mean reduces from $19 \times$ to $12 \times$ ). For latnrm, we consider that the MATLAB code does not use appropriate idiomatic constructs, and that slowdowns the execution in MATLAB. In the case of firld and subband, we are already using MATLAB built-in functions (e.g., sum), and the impact of pre-allocation does not completely explains the speedups (firld reduces to $63 \times$; subband increases to $33 \times$ ). The speedups come from the transformation presented in Section 4.2. We did an experiment where we implemented our own MATLAB version of sum, and used it to generate $\mathrm{C}$ code instead of doing the transformation. In this case, the $s u b$ band speedup was reduced to $2 \times$ (MATISSE could not generate $\mathrm{C}$ code using the custom sum for the other benchmarks where sum is used, such as firld).

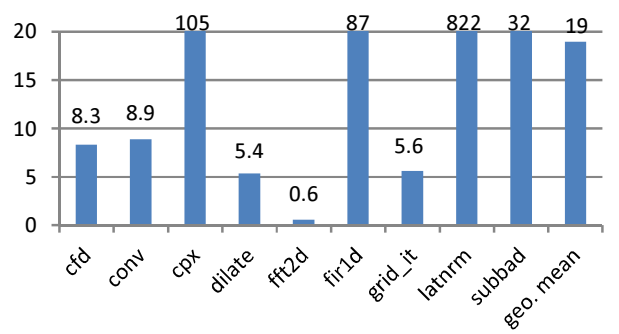

Figure 9. Speedups when comparing the execution of MATLAB code with the $\mathrm{C}$ code generated by MATISSE.

Table 2 shows the speedups achieved by the code generated with the OpenCL engine, when compared with the execution of the original MATLAB code, running on MATLAB, and the C version generated by MATISSE without OpenCL directives. From the set of benchmarks, we chose those whose loops could be parallelized and currently supported by the OpenCL generator. The OpenACC based pragmas were manually added to the MATLAB code. The generated OpenCL code run in the nVidia Quadro NVS-290 of the desktop PC.

We achieved a speedup of $1.37 \times$ on dilate over C-only code, after increasing the size of the inputs (from a $512 \times 512$ image to $1024 \times 1024)$. For $c p x$ and subband, the OpenCL implementation could not beat the pure $\mathrm{C}$ code currently generated by MATISSE. These results were expected as our current OpenCL generator is not able to take fully advantage of the target GPUs.

Table 2. Speedup of $\mathrm{C}+$ OpenCL compared with execution in MATLAB and execution of C-only MATISSE on Core 2 Duo.

\begin{tabular}{|l|l|l|}
\hline \multicolumn{1}{|c|}{ Benchmark } & \multicolumn{1}{c|}{ MATLAB } & C-only MATISSE \\
\hline \hline dilate $1024 \times 1024$ & 17.5 & 1.37 \\
\hline cpx & 18.8 & 0.19 \\
\hline subband & 1.9 & 0.05 \\
\hline
\end{tabular}

\section{Related Work}

Given the importance of MATLAB there have been research efforts to improve the execution of JIT MATLAB compilers. A recent example is the compiler presented in [13] which performs function specialization based on the runtime knowledge of the types of the arguments of the functions. Given the widespread of the use of MATLAB to develop embedded systems and the hardware constraints of such systems that precludes the use of a runtime MATLAB environment, an important aspect is the automatic translation of MATLAB programs into equivalent $\mathrm{C}$ code.

DeRose and Padua developed the FALCON environment [14] that translates MATLAB to FORTRAN90 code. They leverage an aggressive use of static and type inference for base types (doubles and complex) as well as shape (or rank) of the matrices. Other researchers have explored the reuse of storage for array variables 
across a MATLAB code thus reducing the memory footprint of the corresponding $\mathrm{C}$ reference code [15]. Joisha et al [16] focused on type and shape inference techniques. Researchers have also relied on a mix of type inference approaches and user's provided information. For instance, [17][18] use annotations to specify data types and shapes and simple type inference analysis and target VHDL code for hardware synthesis onto FPGAs. We specifically note that the focus of our approach is mostly on embedded implementations of the MATLAB programs. In this context, an efficient translation to an implementation language (mainly $\mathrm{C}$ ) is needed. One of the possibilities is to consider a subset of $M A T L A B$ allowing feasible and efficient static compilation. Examples using such a subset are the Matlab Coder [2] and the Embedded Coder [3].

The popularity of the MATLAB language is also reflected in the similar languages that have been proposed. Examples of those languages are Scilab [19] and Octave [20]. A Scilab to C translator [21], named Sci2C, has been developed. Sci2C focus entirely on embedded systems, and is completely dependent on annotations embedded in the Scilab code to specify data sizes and precisions. Our compiler distinguishes from Sci2C as it is able to generate $\mathrm{C}$ code without polluting the original code. Furthermore, Sci2C requires that the size of arrays is fixed and statically known while our compiler also produces $\mathrm{C}$ code when those sizes are unknown. The use of user-specified rules and strategies for code transformations has been used to optimize Octave programs [22]. with loop vectorization and partial evaluation of types and values.

In this work we describe a mechanism for conveying information about types and shape/rank similar in spirit with the notion of Aspects [7]. Previous work has proposed aspect-oriented extensions to MATLAB and an aspect-oriented code transformation language for MATLAB [23]. Other authors have explored aspectoriented approaches for MATLAB [24], but do not use aspects to specify complementary information that can be used by compilers to produce more efficient implementations.

\section{Conclusion}

This paper presented the current status of MATISSE, a compiler infrastructure for MATLAB. MATISSE relies on LARA aspects for specifying data types, shapes, and code instrumentation and specialization, and on the C-IR for type inference and C code generation. We presented the general flow of the tool, and described the possible transformations that can be applied and the optimizations performed by the compiler. The experiments reveal promising performance results, achieving a geometric mean speedup of $12 \times$ over execution in MATLAB when considering 9 benchmarks. Additionally, we described our first steps on OpenCL generation from MATLAB. Our OpenCL generator takes advantage of OpenACC-based directives to decide about the parallelization and about the MATLAB code sections to be mapped to the accelerator. Ongoing work is focused on further optimizing the $\mathrm{C}$ generator and on evaluating and optimizing the OpenCL generator.

\section{Acknowledgments}

This work was partially supported by Fundação para a Ciência e a Tecnologia (FCT) under FEDER/ON2 and FCT project NORTE-07-124-FEDER-000062.

\section{References}

[1] MATLAB - the Language of Technical Computing, http://www.mathworks.com/products/matlab
[2] MATLAB Coder: Generate $\mathrm{C}$ and $\mathrm{C}++$ code from MATLAB code, (C) 2012 The MathWorks, Inc.

[3] Embedded Coder: Generate $\mathrm{C}$ and $\mathrm{C}++$ code optimized for embedded systems, (C) 2014 The MathWorks, Inc.

[4] The OpenCL Specification, Version: 1.0, Doc. Rev.: 48, Khronos OpenCL Working Group, Editor: Aaftab Munshi, Last Rev. Date: $10 / 6 / 09$.

[5] T. S. Czajkowski, et al., "From opencl to high-performance hardware on FPGAs," 22nd Int'l Conf. on Field Progr. Logic and Applications (FPL'12), Oslo, Norway, Aug. 29-31, 2012, pp. 531-534.

[6] J. Bispo, et al., "The MATISSE MATLAB Compiler - A MATrix(MATLAB)-aware compiler InfraStructure for embedded computing SystEms," in IEEE Int'l Conf. on Industrial Informatics (INDIN'13), Bochum, Germany, 29-31 July 2013, pp. 602-608.

[7] G. Kiczales, et al., "Aspect-Oriented Programming," In Proc. European Conference on Object-Oriented Programming (ECOOP'97), Springer-Verlag, LNCS 1241, June 1997, pp. 220-242.

[8] J.D. Gradecki, and N. Lesiecki, Mastering AspectJ: Aspect-Oriented Programming in Java, John Wiley \& Sons, Inc., NY, USA, 2003.

[9] J.M.P. Cardoso, et al., "LARA: An Aspect-Oriented Programming Language for Embedded Systems," in Proc. Int. Conf. on AspectOriented Software Development (AOSD'12), Potsdam, Germany, March 25-30, 2012, pp. 179-190.

[10] J.M.P. Cardoso, P. Diniz, J.G. Coutinho, and Z. Petrov (eds.), Compilation and Synthesis for Embedded Reconfigurable Systems, Springer, May 2013

[11] A. Aho, J. Ullman, M. Lam, and R. Sethi, Compilers: Principles, Techniques and Tools, Addison Wesley, 2006.

[12] The OpenACC ${ }^{\mathrm{TM}}$ Application Program Interface, August 2013. Version: 2.0a, (C) 2011-2013 OpenACC-Standard.org.

[13] M. Chevalier-Boisvert, L. Hendren, and C. Verbrugge, "Optimizing MATLAB through Just-In-Time Specialization," in Int. Conf. on Compiler Construction (CC'10), March 2010, pp. 46-65.

[14] L. De Rose, and D. Padua, "Techniques for the Translation of MATLAB programs into Fortran 90," in ACM Trans. Program. Lang. Syst., 21, 2 (Mar. 1999), pp. 286-23.

[15] P. Joisha, and P. Banerjee, "Static array storage optimization in MATLAB", in Proc. ACM Conf. on Prog. Language Design and Implementation (PLDI'03), June 9-11, 2003, San Diego, CA, USA, pp. 258-268.

[16] P. Joisha, and P. Banerjee, "An algebraic array shape inference system for MATLAB," in ACM TOPLAS, 2006; 28(5), pp. 848907.

[17] A. Navak, M. Haldar, A. Choudhary, and P. Banerjee, "Parallelization of MATLAB Applications for a Multi-FPGA System", in Proc. 9th IEEE Symp. on Field-Programmable Custom Computing Machines (FCCM'01), Rohnert Park, CA, USA, May, 2001, pp. 1-9.

[18] P. Banerjee, at al., "Automatic Conversion of Floating Point MATLAB Programs", in Proc. 11th IEEE Symp. on FieldProgrammable Custom Computing Machines (FCCM'03), Napa, CA, USA, 2003.

[19] Scilab, http://www.scilab.org/

[20] The Octave Home Page. http://www.gnu.org/software/octave/

[21] Scilab 2 C - Translate Scilab code into C code, http://forge.scilab.org/index.php/p/scilab2c/

[22] K. Olmos, and E. Visser, "Turning dynamic typing into static typing by program specialization in a compiler front-end for Octave," in Proc. 3rd IEEE Int. Workshop on Source Code Analysis and Manipulation (SCAM'03), 26-27 Sept. 2003, pp. 141-150.

[23] J.M.P. Cardoso, et al., "A Domain-Specific Aspect Language for Transforming MATLAB Programs," in Domain-Specific Aspect Language Workshop (DSAL'2010), part of AOSD'2010, March 1519, 2010, Rennes \& Saint Malo, France.

[24] T. Aslam, J. Doherty, A. Dubrau, and L. Hendren, "AspectMatlab: An Aspect-Oriented Scientific Programming Language", in Proc. Aspect Oriented Software Development Conference (AOSD), March 2010, ACM, NY, USA, pp. 181-192. 